Special issue of the 2nd International Conference on Computational and Experimental Science and Engineering (ICCESEN 2015)

\title{
Unsupervised Method for Building Detection using Gabor Filters
}

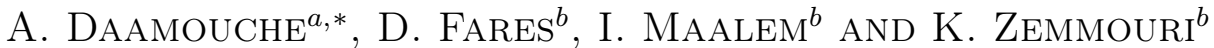 \\ ${ }^{a}$ M'hamed Bougara University, Electronics Department, Boumerdes, Algeria \\ ${ }^{b}$ M'hamed Bougara University, IGEE, Boumerdes, Algeria
}

\begin{abstract}
Automatic methods are a convenient solution to extract buildings from remote sensing images. In this paper, we propose an unsupervised method to extract buildings by means of the Gabor filters and morphological operators. Our proposed method starts from a set of the Gabor filter parameters which is selected empirically to generate a two-dimensional impulse response. The convolution of the impulse response with the image of interest enhances the buildings and attenuates the background. Moreover, morphological operators are applied to further refine the resulting class of interest. The developed algorithm is applied on many remotely sensed images; the obtained results are highly satisfactory and promising.
\end{abstract}

DOI: 10.12693/APhysPolA.130.28

PACS/topics: 07.05.Pj, 42.30.Sy, 42.68.Wt

\section{Introduction}

The Gabor filter is one of the most used filters in both signal and image processing; it was first introduced by Gabor in 1946. The motivation to use the Gabor functions is mostly biological, since Gabor-like receptive fields have been found in the visual cortex. (Frequency and orientation representations of Gabor filters are similar to those of the human visual system.) The Gabor functions act as low-level oriented edge and texture discriminators and are sensitive to different frequencies and scale information. These facts raised considerable interest and motivated researchers to extensively exploit the properties of Gabor functions.

Many authors made use of thresholding to extract buildings from images [1, 2]. In the works of [3] and [4], the authors proposed automatic thresholding. We propose in this paper an unsupervised method to extract buildings using the Gabor filters and morphological operators.

Gabor-filter-based methods have been successfully applied for a variety of machine vision applications [5], such as edge detection, object detection [6], texture segmentation [7], image classification, fingerprint and face recognition.

\section{2D Gabor filter}

Gabor was interested in the Gabor expansion for one-dimensional signals, whereas since 1980, the twodimensional (2D) versions of the elementary functions (called 2D Gabor filters) have been extensively used as convolution filters, which was motivated by the research results in biological vision systems. In 1980, Daugman

*corresponding author; e-mail: ahdaamouche@yahoo.com proposed 2D Gabor filters for modeling simple receptive fields in striate cortex.

The 2D Gabor filter is our concern in this paper because it is often used in image processing as images are 2D signals. The 2D Gabor function is a 2D sinusoidal carrier multiplied by a Gaussian envelope. In general, the 2D Gabor filter has real and imaginary components representing orthogonal directions. The two components may be formed into a complex number or used individually.

The Gabor filter we are going to use in our work is given by the following formula [6]:

$$
\begin{aligned}
& h(x, y)=\exp \left(-\frac{1}{2}\left(\frac{x^{2}}{\sigma_{x}^{2}}+\frac{y^{2}}{\sigma_{y}^{2}}\right)\right) \\
& \times \cos \left(2 \pi \mu_{0} x+\varphi\right),
\end{aligned}
$$

where $\mu_{0}$ denotes the radial frequency of the filter; $\sigma_{x}$ and $\sigma_{y}$ represent the space constants of the filter. The filter corresponding to Eq. (1) is real valued, even symmetric and oriented along the horizontal $x$-axis.

\section{The proposed method}

The proposed method starts from the selection of the appropriate Gabor function; in this paper we adopted the function given by Eq. (1) because of its effectiveness to separate the class of interest from the background. This feature extraction is the most important step in the proposed method since the remaining steps depend totally on the success of it. After selecting the Gabor function, one should find the set of parameters which well suits the image at hand. So far, there is no available method which finds the optimal solution to this problem. Thus, we rely on empirical method and we use visual inspection to tune the Gabor function parameters until we arrive at a satisfactory choice. The results after thresholding are refined by means of mathematical operators [8]. The proposed method is summarized as the block diagram in Fig. 1. 


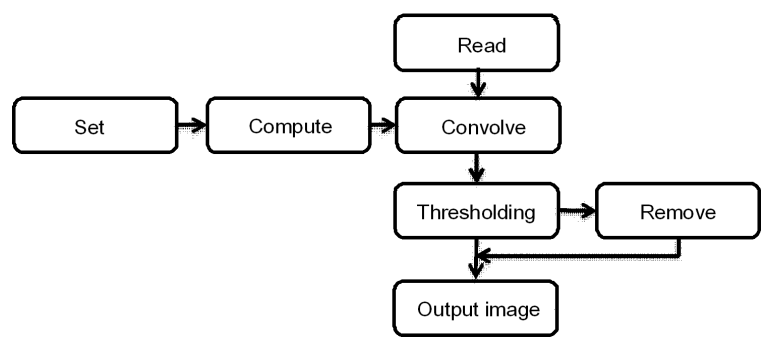

Fig. 1. Block diagram of the proposed method.

\section{Experimental results}

For the sake of the assessment of the proposed algorithm, we used a high resolution image shown in Fig. 2. The parameter set which gives the best results is $\left\{\sigma_{x}=3, \sigma_{y}=2, \phi=27, u_{0}=\pi / 4\right\}$. By best results we mean that most of buildings are isolated from the remaining objects.

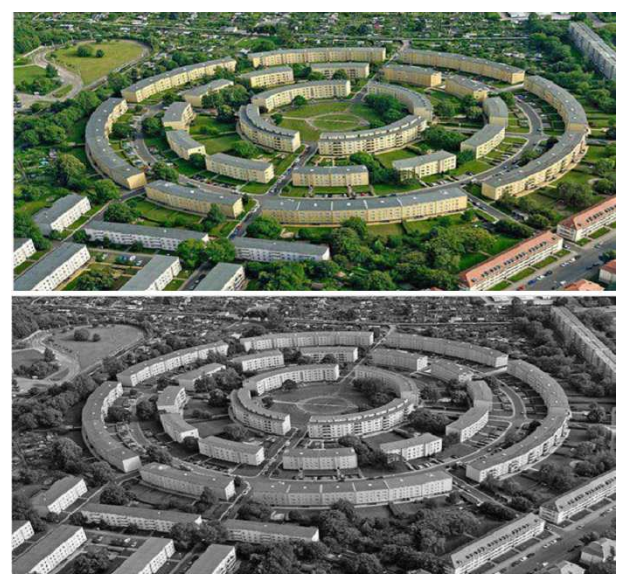

Fig. 2. The original true color image (top) and its grey image (bottom).

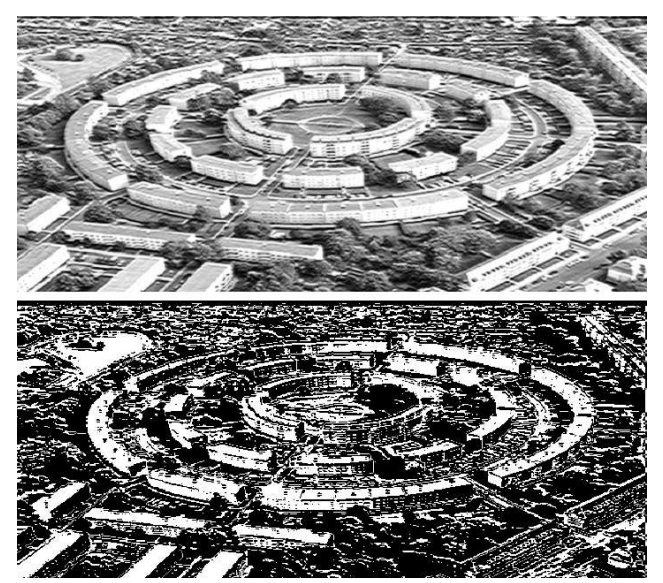

Fig. 3. The results using the set $\left(\sigma_{x}=3, \sigma_{y}=2\right.$, $\left.\phi=27, u_{0}=\pi / 4\right)$ : (a) the image after convolution;

(b) the resulting image after thresholding.

Figure 3 shows the result of applying the Gabor filter on the image using the set $\left\{\sigma_{x}=3, \sigma_{y}=1, \phi=27, u_{0}=5 \pi / 4\right\}$; Fig. 3a is the image after convolution. Thresholding is applied to extract the buildings with threshold values $T 1=0.6$ and $T 2=1.45$; the corresponding binary image is shown in Fig. $3 \mathrm{~b}$.

\subsection{Results refinement}

The binary images resulted from testing the algorithm show some objects that are not parts of the buildings. Figure 4 presents the new binary images after applying morphological operators.

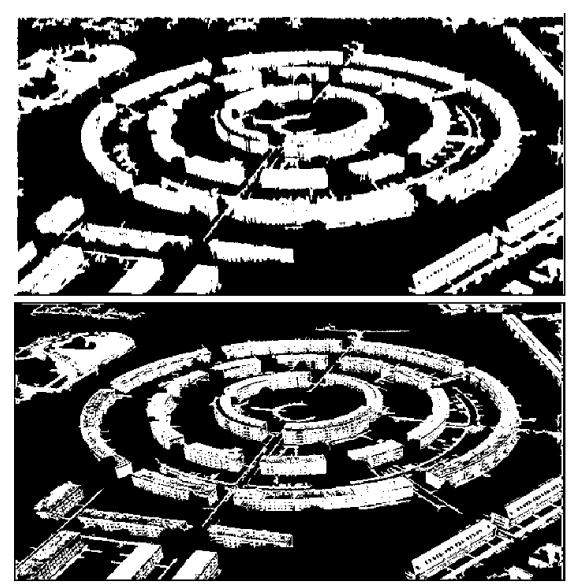

Fig. 4. The refined images after removing small objects by means of morphological operators.

\section{Conclusion}

In this paper, we presented an algorithm for building detection using the Gabor filters. The experimental task was performed on remotely sensed image. The algorithm is developed so that the building features in an image can be extracted. The conducted experiments showed very acceptable results in which the buildings were mostly detected.

For further work, we will focus on adaptive methods for finding the optimal set of parameters and explore more Gabor function types.

\section{References}

[1] A. Stassopoulou, T. Caelli, Int. J. Pattern Recog. Artif. Intell. 14, 715 (2000).

[2] R. Irvin, D.M. McKeown, IEEE Trans. Syst. Man Cybern. 19, 1564 (1989).

[3] J.M. Scanlan, D.M. Chabries, R. Christiansen, IEEE Trans. Acoust. Speech Signal Process. 4, 2057 (1990).

[4] J.D. Tsai, in: Proc. IEEE Int. Geoscience and Remote Sensing Symp., 2003, p. 732.

[5] T.S. Lee, IEEE Trans. Pat. Anal. Mach. Intell. 18, 959 (1996).

[6] A.K. Jaın, T. Nalını, K. Ratha, S. Lakshmanan, Pattern Recogn. 30, 295 (1997).

[7] U. Marmol, Arch. Photogram. Cartogr. Remote Sens. 22, 325 (2011).

[8] W.P. Soille, M. Pesresi, IEEE Trans. Geosci. Remote Sens. 40, 2042 (2002). 